\title{
Article \\ Higher Radiation Dose to the Immune Cells Correlates with Worse Tumor Control and Overall Survival in Patients with Stage III NSCLC: A Secondary Analysis of RTOG0617
}

\author{
Jian-Yue Jin ${ }^{1}$, Chen $\mathrm{Hu}^{2,3}$, Ying Xiao ${ }^{4}$, Hong Zhang ${ }^{5}$, Rebecca Paulus ${ }^{2}$, Susannah G. Ellsworth ${ }^{6}$, \\ Steven E. Schild ${ }^{7}$, Jeffrey A. Bogart ${ }^{8}$, Michael Chris Dobelbower ${ }^{9}$, Vivek S. Kavadi ${ }^{10}$, Samir Narayan ${ }^{11}$, \\ Puneeth Iyengar ${ }^{12}$, Cliff Robinson ${ }^{13}$, Joel S. Greenberger ${ }^{6}$, Christopher Koprowski $^{14}$, Mitchell Machtay ${ }^{15}$, \\ Walter Curran ${ }^{16}$, Hak Choy ${ }^{12}$, Jeffrey D. Bradley ${ }^{13}$ and Feng-Ming (Spring) Kong $17,18, * \mathbb{D}$
}

check for

updates

Citation: Jin, J.-Y.; Hu, C.; Xiao, Y.;

Zhang, H.; Paulus, R.; Ellsworth, S.G.; Schild, S.E.; Bogart, J.A.; Dobelbower, M.C.; Kavadi, V.S.; et al. Higher Radiation Dose to the Immune Cells Correlates with Worse Tumor Control and Overall Survival in Patients with Stage III NSCLC: A Secondary Analysis of RTOG0617. Cancers 2021, 13, 6193. https://doi.org/10.3390/ cancers 13246193

Academic Editors: Meritxell Arenas and Pierfrancesco Franco

Received: 1 July 2021

Accepted: 6 August 2021

Published: 8 December 2021

Publisher's Note: MDPI stays neutral with regard to jurisdictional claims in published maps and institutional affiliations.

Copyright: (C) 2021 by the authors. Licensee MDPI, Basel, Switzerland. This article is an open access article distributed under the terms and conditions of the Creative Commons Attribution (CC BY) license (https:// creativecommons.org/licenses/by/ $4.0 /)$.
1 Department of Radiation Oncology, Case Western Reserve University and University Hospitals of Cleveland, Cleveland, OH 44106, USA; Jian-Yue.Jin@UHhospitals.org

2 NRG Oncology Statistics and Data Management Center, Philadelphia, PA 19103, USA;

HuC@NRGOncology.org or huc@jhu.edu (C.H.); paulusr@nrgoncology.org (R.P.)

3 Sidney Kimmel Comprehensive Cancer Center, Johns Hopkins University School of Medicine, Baltimore, MD 21218, USA

4 Abramson Cancer Center, University of Pennsylvania, Philadelphia, PA 19103, USA; Ying.Xiao@uphs.upenn.edu

5 Department of Radiation Oncology, School of Medicine, University of Maryland, Maryland, MD 20742, USA; Hong.Zhang@umm.edu

6 Department of Radiation Oncology, University of Pittsburgh Medical Center, Pittsburgh, PA 16251, USA; ellsworths3@upmc.edu (S.G.E.); greenbergerj@@upmc.edu (J.S.G.)

7 Department of Radiation Oncology, Mayo Clinic Hospital, Phoenix, AZ 85054, USA; sschild@mayo.edu

8 Department of Radiation Oncology, State University of New York Upstate Medical University, Syracuse, NY 13210, USA; bogartj@upstate.edu

9 Department of Radiation Oncology, University of Alabama at Birmingham Cancer Center, Birmingham, AL 35233, USA; mdobelbower@uabmc.edu

10 USON-Texas Oncology-Sugar Land, Sugar Land, TX 77479, USA; vivek.kavadi@usoncology.com

11 Michigan Cancer Research Consortium CCOP, Ann Arbor, MI 48106, USA; Samir.Narayan@stjoeshealth.org

12 Department of Radiation Oncology, University of Texas Southwestern Medical School, Dallas, TX 75235, USA; Puneeth.Iyengar@UTSouthwestern.edu (P.I.); hak.choy@utsouthwestern.edu (H.C.)

13 Department of Radiation Oncology, Washington University in St. Louis, St. Louis, MO 63108, USA; crobinson@radonc.wustl.edu (C.R.); jeffrey.d.bradley@emory.edu (J.D.B.)

14 Christiana Care Health Services, Inc. CCOP, Newark, DE 19718, USA; ckoprowski@christianacare.org

15 Department of Radiation Oncology, Penn State University Cancer Institute, Hershey, PA 17033, USA; mmachtay@pennstatehealth.psu.edu

16 Department of Radiation Oncology, Winship Cancer Institute, Emory University, Atlanta, GA 30322, USA; wcurran@emory.edu

17 Department of Clinical Oncology, Hong Kong University Shenzhen Hospital, Shenzhen 518009, China

18 Department of Clinical Oncology, Queen Mary Hospital, Li Ka Shing Medical School, The University of Hong Kong, Hong Kong 999077, China

* Correspondence: kong0001@hku.hk; Tel.: +852-5393-6858

Simple Summary: Emerging evidence indicates that the immune system plays an important role in controlling tumors during radiotherapy, and radiation-induced immune toxicity such as lymphopenia is associated with poor survival. However, the immune system is not considered as a critical organ at risk in radiotherapy partially because the radiation dose to the immune system is difficult to compute. In this study, we developed a model to compute the radiation dose to the circulating blood, which contains the majority of active immune cells. We then validated this model by examining the correlations of the blood dose with treatment outcome for patients enrolled in the NRG/RTOG0617 phase III clinical trial. We demonstrated that the blood dose was significantly and independently associated with overall survival and local progression-free survival. This result suggests that radiation dose to circulating immune cells is critical for tumor control, and decreasing the dose to the immune system has the potential to improve survival. 


\begin{abstract}
Background: We hypothesized that the Effective radiation Dose to the Immune Cells (EDIC) in circulating blood is a significant factor for the treatment outcome in patients with locally advanced non-small-cell lung cancer (NSCLC). Methods: This is a secondary study of a phase III trial, NRG/RTOG 0617, in patients with stage III NSCLC treated with radiation-based treatment. The EDIC was computed as equivalent uniform dose to the entire blood based on radiation doses to all blood-containing organs, with consideration of blood flow and fractionation effect. The primary endpoint was overall survival (OS), and the secondary endpoints were progression-free survival (PFS) and local progression-free survival (LPFS). The EDIC-survival relationship was analyzed with consideration of clinical significant factors. Results: A total of 456 patients were eligible. The median EDIC values were 5.6 Gy (range, 2.1-12.2 Gy) and 6.3 Gy (2.1-11.6 Gy) for the low- and high-dose groups, respectively. The EDIC was significantly associated with OS (hazard ratio [HR] $=1.12$, $p=0.005)$ and LPFS (HR $=1.09, p=0.02)$ but PFS $(\mathrm{HR}=1.05, p=0.17)$ after adjustment for tumor dose, gross tumor volume and other factors. OS decreased with an increasing EDIC in a non-linear pattern: the two-year OS decreased first with a slope of $8 \% / \mathrm{Gy}$ when the EDIC < 6 Gy, remained relatively unchanged when the EDIC was 6-8 Gy, and followed by a further reduction with a slope of $12 \% / G y$ when the EDIC > 8 Gy. Conclusions: The EDIC is a significant independent risk factor for poor OS and LPFS in RTOG 0617 patients with stage III NSCLC, suggesting that radiation dose to circulating immune cells is critical for tumor control. Organ at risk for the immune system should be considered during RT plan.
\end{abstract}

Keywords: non-small-cell lung cancer; radiotherapy; survival; radiation-induced immune toxicity

\title{
1. Introduction
}

Lung cancer is the leading cause of cancer-related death. Over $85 \%$ of lung cancer cases are non-small-cell lung cancer (NSCLC) [1], 40\% of which are stage III [1,2]. The standard of care for unresectable stage III NSCLC is radiotherapy (RT) and concurrent chemotherapy [3], with recent data supporting the routine integration of adjuvant immunotherapy [4]. Despite advances in RT technology, treatment outcomes remain suboptimal, and local disease progression is a major cause of death [4]. Intensifying local therapy with RT dose escalation was therefore proposed to improve local tumor control and survival [5]. However, RTOG 0617, the largest study to date on dose-response effects in stage III NSCLC, demonstrated significantly worse overall survival (OS) in patients treated on the high-dose arm (74 Gy) vs. the low-dose arm (60 Gy) [6].

Radiation is known for immune modulation effect. Radiation-induced tumor cell killing can activate the immune system through various mechanisms including releasing tumor-specific antigens [7]. Preclinical studies have demonstrated that the immune system plays a key role in tumor control after RT [8,9]. Treatments with RT alone or RT combined with immunotherapy can control tumors in immunocompetent mice, but not in immunedeficient ones [8]. An abscopal effect (i.e., shrinkage of un-irradiated tumors outside the RT field) has been observed in animal studies [8,9] and in clinical settings [10]. While these observations suggest that RT may augment anti-tumor immunity in special situations, RT is also well known for its immunosuppressive effects. One of the most common and clinically significant features of radiation-induced immunosuppression is radiationinduced lymphopenia [11,12], which has been repeatedly reported as a risk factor for poorer survival in several cancers, and a recent pooled analysis reported significance in patients with multiple treatment-refractory solid tumors including NSCLC [11-15].

However, the immune system has not yet been considered as a critical organ at risk for RT planning in practice, though RT dose escalation to tumor is also expected to increase the radiation dose to immune-related structures. We hypothesized that high radiation dose to the immune system may impair various immune functions including anti-tumor immunity, consequently decreasing tumor control. Specifically, we hypothesized that circulating immune cells in blood are an important target for radiation-induced immunosuppression 
because: (1) radiation-induced lymphopenia occurs following breast and brain radiation, where the treated field contains little marrow or lymphatic tissue [16-18]; (2) circulating lymphocytes in blood are one of the most radiosensitive cell types. To test these hypotheses, we first developed a model to compute Effective Dose to Immune Cells in circulating blood (EDIC) as an estimation of equivalent uniform dose to the entire blood during the RT course, and then evaluated the relationship between the EDIC and the risks of local tumor progression and death. The RTOG 0617 trial provided an ideal setting for testing this hypothesis, as detailed dosimetric and survival data were available for nearly all patients enrolled in this large phase III cooperative group trial treated with high and low radiation doses.

\section{Materials and Methods}

\subsection{Clinical and Dosimetric Patient Data}

RTOG 0617 was a phase III trial for unresectable stage III NSCLC. The study details and primary analysis results have been published previously [6]. Briefly, all patients received conformal radiotherapy with concurrent and consolidation chemotherapy (carboplatin and paclitaxel). A two-by-two factorial randomized design was used to assign patients to two different RT dose groups (60 Gy vs. 74 Gy) and systemic therapy arms (carboplatin/paclitaxel with or without cetuximab). Patients included in this secondary analysis must have retrievable RT plans and must have received a confirmed dose of at least $50 \mathrm{~Gy}$, which was an arbitrary cut-off chosen to identify patients who had received a clinically meaningful RT dose. Clinical factors tested for confounding effects included baseline Zubrod performance status, use of positron emission tomography (PET) during staging, tumor histology, age at randomization, gender, race, tumor location(s), weight loss, smoking history, gross tumor volume (GTV), and whether the full course of chemotherapy was received. Conventional radiation dosimetric data such as mean lung dose (MLD), mean heart dose (MHD) and integral total dose volume (ITDV) were included for modeling. ITDV is the integration of dose in the total irradiated volume in the scanned CT image.

\subsection{EDIC Computation}

The immune system is a complex interplay of multiple features and functions of immune cells. Lymphocytes are the most important functional immune cells. Lymphocytes originate in the bone marrow and/or thymus and circulate through the body into various organs via blood vessels, and may return to the blood circulation through lymph nodes and lymphatic ducts. They also circulate through other lymphatic organs such as the spleen. In addition, lymphocytes (specifically T cells) may reside within the tissue of various organs [19]. They even reside within tumor tissue as infiltrated $\mathrm{T}$ cells and play a key role in RT-mediated anti-tumor immunity [19]. In this study, we considered the lymphocytes in circulating blood as a key target for radiation-induced suppression in the anti-tumor immunity because the tumor-resident $T$ cells are very radio-resistant [19], and the lymphocytes in circulating blood provide continuous supply for the loss of tumorresident $\mathrm{T}$ cells during their anti-tumor activities. However, it is very challenging to determine the radiation dose to the lymphocytes in circulating blood because they are moving targets. Using a blood-flow continuity principle, we first calculated the blood dose and volume contributed by a single fraction of radiation to each particular bloodcontaining organ, including lung, heart, great vessels and body mass. The fractionation effect of irradiation on flowing blood through each organ was modelled using a similar approach reported by Yovino et al. [18]. The blood dose volume was then converted into an equivalent uniform dose (EUD). The total effective blood dose was the sum of the EDUs from the contributions of all irradiated organs. We defined this sum of EUDs as the EDIC, the Effective Dose to the Immune Cells in circulating blood. This EDIC model was presented in the 2017 ASTRO annual meeting [20]. A detailed description of the approach to EDIC derivation is described in the Appendix A. The EDIC is finally expressed as the following equation for patients receiving $\geq 25$ fractions of thoracic radiation: 


$$
E D I C=B_{1} \% * M L D+B_{2} \% * M H D+\left[B_{3} \%+B_{4} \% * k_{1} *\left(\frac{n}{45}\right)^{\frac{1}{2}}\right] * \operatorname{ITDV} /\left(61.8 * 10^{3}\right)
$$

where $B_{1} \%=0.12, B_{2} \%=0.08, B_{3} \%=0.45$ and $B_{4} \%=0.35$ represent the percentages of blood volume within the four major blood-containing organs (lung, heart, great vessels, and small vessels / capillaries in all other organs, respectively) out of the total blood volume in the body; MLD, MHD and ITDV are the mean lung dose, mean heart dose and integral total dose volume; $k_{1}=0.85$ is a dose effectiveness factor due to the small percentage of cardiac output for the small vessels/capillaries; and $61.8 \times 10^{3}\left(\mathrm{~cm}^{3}\right)$ is the average total body volume, assuming average weight of $63 \mathrm{~kg}(140 \mathrm{lbs})$ and density of $1.02 \mathrm{~g} / \mathrm{cm}^{3}$.

\subsection{Outcomes and Statistical Considerations}

The primary endpoint was overall survival (OS). The progression-free survival (PFS) and local progression-free survival (LPFS) were tested as secondary endpoints. These endpoints were analyzed as time-to-event data and calculated from the date of randomization to the date of respective event or last follow-up. The OS event was death due to any cause; the PFS event was the first occurrence of any progression or death; and the LPFS event was the first occurrence of local failure or death. These rates were estimated using the Kaplan-Meier method, and the distributions between different groups were compared using the log-rank test. Cox proportional hazards models were used to evaluate the relationships between the EDIC and other factors with OS, PFS and LPFS. Because the EDIC was derived from the combination of MLD, MHD and ITDV, these variables were evaluated individually under multivariable analyses to avoid potential collinearity. The tumor dose effect was adjusted by stratification in both univariate and multivariate analyses. The functional forms of the EDIC in the Cox models were explored both linearly and using restricted cubic splines [21]. To illustrate the non-linear functional form of the EDIC in the Cox model, the EDIC was also categorized based on quartiles and absolute EDIC values. The proportionality assumption was graphically assessed using plots of $\log (-\log [$ survival] $)$ versus log of survival time, and tested using a formal test based on the Schoenfeld residuals [22]. Interaction terms (e.g., potentially differential effects of the EDIC on outcomes by different levels of patient characteristics) were also examined using the Wald test.

All statistical tests were two-sided and performed using SAS 9.4 software (SAS Institute, Cary, NC, USA) and R version 3.4.0 (available at https: / / www.r-project.org / (accessed on 11 July 2017). $p<0.05$ was considered statistically significant.

\section{Results}

\subsection{Patient Characteristics and the EDIC}

Of 495 patients enrolled in RTOG 0617, 29 were excluded due to failure of RT plan retrieval. Ten additional patients were excluded due to errors in plan archiving or other reasons: RT plans missed the target $(n=1)$, wrong RT plans were archived (exact same plans for 2 different patients; $n=4)$, or the total dose received was $\leq 50 \mathrm{~Gy}(n=5)$. Of the 456 eligible patients, 256 and 200 were originally assigned to the standard (60 Gy) and highdose arms (74 Gy), respectively; 261 patients received $60 \mathrm{~Gy}$ (including some originally assigned to the high-dose arm), 165 received 74 Gy, 4 received 52-58 Gy, 12 received 62-66 Gy, and 6 received 67-72 Gy. Patients were categorized according to the actual dose received, with a high dose defined as $\geq 67 \mathrm{~Gy}$. Based on this definition, 285 and 171 patients were placed into the low- and high-dose groups, respectively.

The median follow-up time for patients alive at the last evaluation was 30.3 months (range, 2.5-61.5 months). Demographic, clinical, and dosimetric data for the included patients are summarized in Table S1.

The EDIC was calculated for all 456 patients. The median EDIC values were 5.6 Gy (range, 2.1-12.2 Gy) for the low-dose group, $6.3 \mathrm{~Gy}(2.1-11.6 \mathrm{~Gy})$ for the high-dose group, and 5.9 Gy (2.1-12.2 Gy) for all patients. 


\subsection{Univariate Analysis of the EDIC and Clinical Factors for OS, PFS and LPFS}

EDIC was significantly associated with OS, PFS and LPFS (Table 1) after adjusting for the tumor dose effect using stratification. Similar to previously reported results, gender, Zubrod performance status, tumor histology, smoking history, use of PET staging, and American Joint Committee on Cancer (AJCC) stage were not significantly associated with OS, PFS and LPFS, whereas the occurrence of grade $\geq 3$ esophagitis/dysphagia and completion of the full course of chemotherapy were significantly associated with OS, PFS and LPFS (Table 1). According to the dose that the patients actually received, the low-dose patients had marginally better OS $(p=0.10)$ and PFS $(p=0.08)$, and significantly better LPFS ( $p=0.02)$ than the high-dose patients. Tumor location (central/lower left lobe vs. others) was significantly associated with OS only. Interestingly, all dosimetric factors, including GTV, MLD, and MHD, were significantly associated with OS, PFS and LPFS; ITDV was significant for OS but PFS and LPFS (Table 1).

Table 1. Univariate analysis with stratification of patients by actual received RT dose *.

\begin{tabular}{|c|c|c|c|c|c|c|}
\hline \multirow[t]{2}{*}{ Variables } & \multicolumn{2}{|l|}{ OS } & \multicolumn{2}{|l|}{ PFS } & \multicolumn{2}{|l|}{ LPFS } \\
\hline & HR $(95 \%$ CI) & $p$ & HR $(95 \%$ CI) & $p$ & HR (95\% CI) & $p$ \\
\hline Prescription dose: cont. & $1.31(1.04,1.67)$ & 0.01 & $1.22(0.98,1.51)$ & 0.07 & $1.34(1.07-1.67)$ & 0.01 \\
\hline Actual received dose: cont. & $1.22(0.95,1.56)$ & 0.10 & $1.21(0.98,1.50)$ & 0.08 & $1.32(1.05,1.65)$ & 0.017 \\
\hline Age: cont. & $1.01(0.99,1.02)$ & 0.24 & $0.997(0.99,1.009)$ & 0.59 & $1.01(0.99,1.02)$ & 0.26 \\
\hline Gender: Male (RL) vs. Female & $0.83(0.65,1.06)$ & 0.13 & $0.96(0.77,1.19)$ & 0.71 & $0.88(0.70,1.10)$ & 0.27 \\
\hline Zubrod status: 0 (RL) vs. 1 & $1.02(0.80,1.30)$ & 0.86 & $0.95(0.76,1.18)$ & 0.64 & $1.01(0.81,1.27)$ & 0.91 \\
\hline Histology: Non-Sq (RL) vs. Sq & $1.13(0.88,1.43)$ & 0.34 & $1.02(0.82,1.26)$ & 0.87 & $1.19(0.95,1.49)$ & 0.12 \\
\hline Smoking history: Yes (RL) vs. No & $0.72(0.43,1.21)$ & 0.22 & $0.79(0.50,1.26)$ & 0.32 & $0.80(0.50,1.30)$ & 0.37 \\
\hline RT technique: 3D (RL) vs. IMRT & $0.89(0.70,1.13)$ & 0.33 & $1.04(0.84,1.28)$ & 0.74 & $1.06(0.84,1.32)$ & 0.64 \\
\hline PET staging: No (RL) vs. Yes & $0.76(0.52,1.11)$ & 0.16 & $0.87(0.61,1.24)$ & 0.45 & $0.83(0.58,1.21)$ & 0.34 \\
\hline AJCC stage: IIIA (RL) vs. IIIB & $1.03(0.80,1.32)$ & 0.82 & $1.08(0.86,1.35)$ & 0.52 & $1.08(0.86,1.37)$ & 0.49 \\
\hline $\begin{array}{l}\text { Tumor location: Not LLL/central } \\
\text { (RL) vs. LLL/central }\end{array}$ & $1.49(1.06,2.09)$ & 0.02 & $1.21(0.88,1.66)$ & 0.25 & $1.33(0.95,1.84)$ & 0.09 \\
\hline Esophagitis grade: $<3$ (RL) vs. $\geq 3$ & $1.77(1.30,2.41)$ & 0.0003 & $1.72(1.29,2.28)$ & 0.0002 & $1.53(1 / 14.2 .06)$ & 0.005 \\
\hline Received full chemo: No (RL) vs. Yes & $0.64(0.46,0.90)$ & 0.009 & $0.72(0.53,0.97)$ & 0.03 & $0.70(0.51,0.97)$ & 0.03 \\
\hline GTV: cont. & $1.21(1.07,1.38)$ & 0.0026 & $1.13(1.01,1.26)$ & 0.03 & $1.13(1.01,1.27)$ & 0.04 \\
\hline Mean lung dose: cont. & $1.05(1.02,1.09)$ & 0.0004 & $1.04(1.01,1.07)$ & 0.003 & $1.03(1.004,1.06)$ & 0.02 \\
\hline Mean heart dose: cont. & $1.02(1.01,1.03)$ & $<0.0001$ & $1.01(1.003,1.02)$ & 0.004 & $1.02(1.007,1.03)$ & 0.0007 \\
\hline Integral total dose: cont. & $1.003(1.001,1.005)$ & 0.0004 & $1.001(1.00,1.003)$ & 0.11 & $1.002(1.00,1.003)$ & 0.03 \\
\hline EDIC: cont. & $1.18(1.10,1.26)$ & $<0.0001$ & $1.10(1.03,1.16)$ & 0.002 & $1.11(1.05,1.18)$ & 0.0009 \\
\hline
\end{tabular}

* The effect of actual received dose has been stratified for all other factors in this univariate analysis except for the prescription dose and actual received dose. Abbreviations: cont., continuous variable; RL, reference level; Sq, squamous; LLL, left lower lobe; RT, radiotherapy; OS, overall survival; PFS, progression-free survival; LPFS, local progression-free survival; HR, hazard ratio; CI, confidence interval; GTV, gross tumor volume; EDIC, Effective Dose to Immune Cells.

\subsection{The EDIC in Multivariable Analysis of Factors Associated with OS, PFS and LPFS}

The EDIC effects were assessed under two different multivariable models: one without the EDIC but with MLD/MHD/ITDV and one with the EDIC but without $\mathrm{MLD} / \mathrm{MHD} / \mathrm{ITDV}$, as there were apparent correlations between MLD/MHD/ITDV and the EDIC. Other clinical and dosimetric factors, including tumor location, gross tumor volume, esophagitis grade, and received full chemo, were considered for these multivariable analyses. The tumor dose effect was adjusted by stratification according to the actual received radiation dose. The occurrence of grade $\geq 3$ esophagitis/dysphagia and completion of chemotherapy were significant for OS, PFS and LPFS in both models (Table 2a-c). Interestingly, in the multivariable Cox model of OS without the EDIC, only GTV was significant but not MHD, MHD and ITDV. On the other hand, in the multivariable Cox model of OS with the EDIC, the EDIC was significant, while GTV was not (Table 2a). 
Table 2. Stratified multivariable analyses with stratification of patients according to the actual received RT dose.

\begin{tabular}{|c|c|c|c|c|}
\hline \multicolumn{5}{|c|}{ (a) } \\
\hline \multirow{2}{*}{ Variables } & \multicolumn{2}{|c|}{ OS without EDIC } & \multicolumn{2}{|c|}{ OS with EDIC } \\
\hline & HR (95\% CI) & $p$ & HR $(95 \%$ CI) & $p$ \\
\hline Tumor location & $1.42(0.98,2.05)$ & 0.07 & $1.41(0.98,2.02)$ & 0.07 \\
\hline Gross tumor volume & $1.16(1.00,1.34)$ & 0.05 & $1.12(0.98,1.28)$ & 0.09 \\
\hline Esophagitis grade & $1.53(1.11,2.11)$ & 0.01 & $1.52(1.10,2.10)$ & 0.012 \\
\hline Received full chemo & $0.58(0.41,0.81)$ & 0.0015 & $0.59(0.42,0.83)$ & 0.003 \\
\hline Mean lung dose & $1.03(0.998,1.070)$ & 0.07 & & \\
\hline Mean heart dose & $1.008(0.995,1.022)$ & 0.21 & & \\
\hline Integral total dose & $1.000(0.998,1.002)$ & 0.93 & & \\
\hline \multirow[t]{2}{*}{ EDIC } & & & $1.12(1.03,1.21)$ & 0.005 \\
\hline & \multicolumn{2}{|c|}{ (b) } & & \\
\hline \multirow{2}{*}{ Variables } & \multicolumn{2}{|c|}{ PFS without EDIC } & \multicolumn{2}{|c|}{ PFS with EDIC } \\
\hline & HR (95\% CI) & $p$ & HR (95\% CI) & $p$ \\
\hline Tumor location & $1.19(0.84,1.68)$ & 0.33 & $1.20(0.85,1.68)$ & 0.30 \\
\hline Gross tumor volume & $1.15(1.01,1.32)$ & 0.04 & $1.08(0.96,1.21)$ & 0.20 \\
\hline Esophagitis grade & $1.64(1.22,2.21)$ & 0.001 & $1.60(1.19,2.15)$ & 0.002 \\
\hline Received full chemo & $0.63(0.46,0.86)$ & 0.003 & $0.66(0.49,0.90)$ & 0.009 \\
\hline Mean lung dose & $1.04(1.006,1.071)$ & 0.02 & & \\
\hline Mean heart dose & $1.005(0.992,1.017)$ & 0.47 & & \\
\hline Integral total dose & $0.998(0.996,1.000)$ & 0.10 & & \\
\hline \multirow[t]{2}{*}{ EDIC } & & & $1.05(0.98,1.12)$ & 0.17 \\
\hline & & & & \\
\hline \multirow{2}{*}{ Variables } & \multicolumn{2}{|c|}{ LPFS without EDIC } & \multicolumn{2}{|c|}{ LPFS with EDIC } \\
\hline & HR $(95 \%$ CI) & $p$ & HR $(95 \%$ CI) & $p$ \\
\hline Gross tumor volume & $1.10(0.96,1.26)$ & 0.16 & $1.07(0.95,1.20)$ & 0.29 \\
\hline Esophagitis grade & $1.36(1.00,1.85)$ & 0.05 & $1.37(1.00,1.86)$ & 0.05 \\
\hline Received full chemo & $0.66(0.48,0.91)$ & 0.012 & $0.67(0.48,0.92)$ & 0.013 \\
\hline Mean lung dose & $1.01(0.98,1.04)$ & 0.48 & & \\
\hline Mean heart dose & $1.012(1.000,1.024)$ & 0.044 & & \\
\hline Integral total dose & $1.00(0.998,1.002)$ & 0.81 & & \\
\hline EDIC & & & $1.09(1.01,1.16)$ & 0.02 \\
\hline
\end{tabular}

Abbreviations: OS, overall survival; $\mathrm{HR}$, hazard ratio; CI, confidence interval; EDIC, effective dose to immune cells; PFS, progression-free survival; LPFS, local progression-free survival.

However, the multivariable PFS model was quite different from the OS model (Table 2b). Both MLD and GTV were significantly associated with PFS in the model without the EDIC, while both GTV and the EDIC were not significant in the model with the EDIC. The multivariable LPFS model was similar to the OS model (Table 2c). The EDIC was a significant factor for LPFS while GTV was not in the model with the EDIC. However, MHD was a significant factor while MLD and GTV were not in the LPFS model without the EDIC.

\subsection{Non-Linear Relationship between the EDIC and OS}

To further analyze the relationship between the EDIC and OS, 456 patients were divided into four groups with equal number of patients per the quartiles of the EDIC (Figure 1a), as well as into six groups with an equal dose increment of 1.5 Gy between two groups (Figure 1b). The Kaplan-Meier curves shown in Figure 1 depict a strong inverse relationship between OS and the EDIC (i.e., the greater the EDIC, the worse the OS). However, the relationship between the EDIC and OS was not strictly linear, because the survival estimates of some intermediate EDIC groups overlap in both Figure 1a,b. To 
further illustrate this non-linear relationship, we estimated the hazard of the EDIC by nonparametric smoothing using restricted cubic splines in a univariate Cox regression model of OS, stratified tumor dose (Figure 2a). This analysis showed that hazard rates increased with increasing EDIC when the EDIC was less than $6.0 \mathrm{~Gy}$ or larger than 8.0 Gy but not between 6 and $8 \mathrm{~Gy}$. This Cox regression analysis showed that the risk of death increased by $23 \% / \mathrm{Gy}(\mathrm{HR}=1.23,95 \% \mathrm{CI}: 1.07-1.41 ; p=0.003)$ with increasing EDIC when the EDIC < 6.0 and by $37 \% / \mathrm{Gy}(\mathrm{HR}=1.37,95 \%$ CI: $1.14-1.64 ; p=0.0007)$ when the EDIC > 8 Gy. However, this curve was relatively flat for EDIC values in the range of 6.0-8.0 Gy.

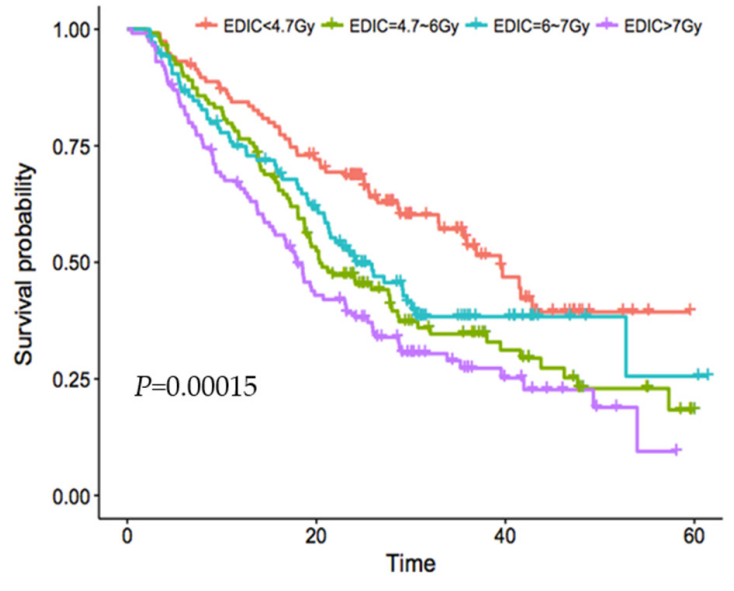

(a)

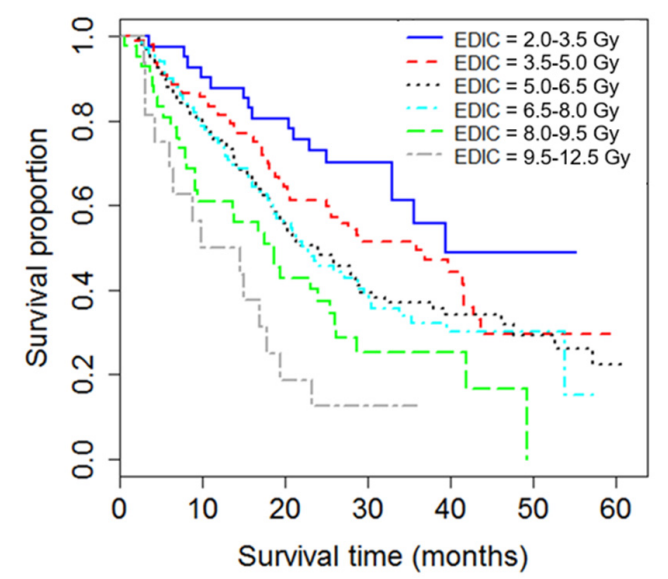

(b)

Figure 1. Overall survival curves separated by the effective dose to immune cells (EDIC). (a) for patients divided into 4 quartiles according to the EDIC); and (b) for patients divided into 6 EDIC groups with a 1.5 Gy dose increment. Survival improved significantly with a reduction in the EDIC. However, overall survival (OS) were not significantly different among patients with an EDIC between 6.0 and $8.0 \mathrm{~Gy}$.

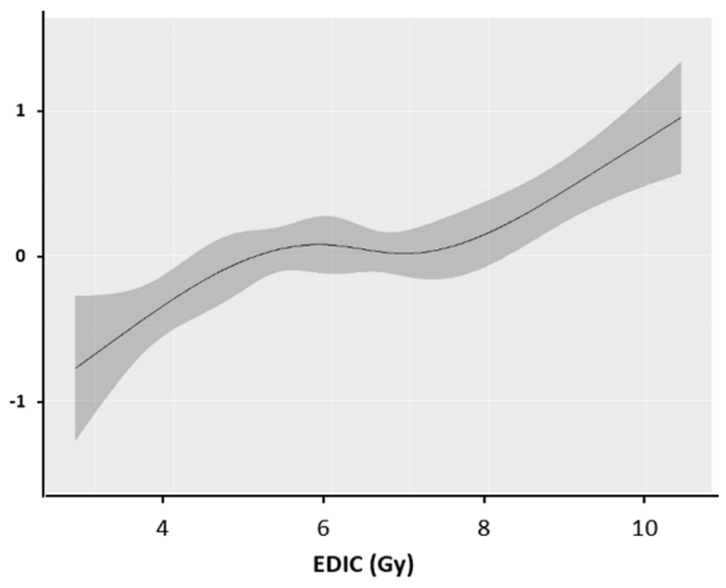

(a)

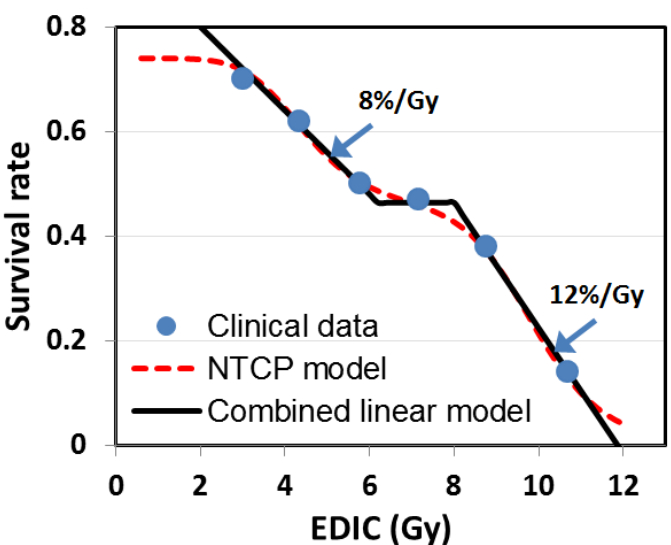

(b)

Figure 2. Quantitative effect of the effective dose to immune cells (EDIC) on the risk of death. (a) Relationship between relative hazard of death and the EDIC. The hazard of death increased with increasing EDIC when EDIC values was $<5.5$ Gy and remained relatively unchanged when EDIC values ranged 5.5 to $7.5 \mathrm{~Gy}$, and again increased with EDIC values > 7.5 Gy. (b) Relationship between 2 year overall survival (OS) rate and the EDIC by a normal tissue complication probability (NTCP) survival model. The clinical data were well fitted by the NTCP model composed of two components, with D50 being 4.5 and $9.9 \mathrm{~Gy}$, respectively. The clinical data could also be described by a combined linear model with 3 parts: (1) for an EDIC < 6.0 Gy, 2 year OS decreased with increasing EDIC at a slope of 8\%/Gy; (2) for an EDIC between 6.0 and 8.0 Gy, 2 year OS remained unchanged; and (3) for an EDIC > 8.0 Gy, 2 year OS decreased with increasing EDIC at a slope of 12\%/Gy. 
This non-linear relationship was also illustrated by the survival dose-response curve for 2 year OS versus the EDIC (Figure $2 b$ ). The six data points were determined from the data of the six subgroups in Figure 1b, with the horizontal axis of each point being the average EDIC for the corresponding subgroup. The data were well fitted by an OS model composed of two normal tissue complication probability (NTCP) components:

$$
O S=0.74 *\left[1-\frac{0.39}{1+\left(\frac{4.5}{E D I C}\right)^{6}}\right] *\left[1-\frac{1}{1+\left(\frac{9.9}{E D I C}\right)^{12}}\right]
$$

with $\mathrm{D}_{50}$ being 4.5 and $9.9 \mathrm{~Gy}$, respectively, for the two NTCP components. Alternatively, the survival dose-response can also be described by a combined linear model in three parts: (1) for an EDIC < 6.0 Gy, 2 year OS decreases with increasing EDIC at a slope of 8\%/Gy; (2) for an EDIC of 6.0-8.0 Gy, OS does not vary; and (3) for an EDIC > 8.0 Gy, 2 year OS decreases with increasing EDIC at a slope of $12 \% / \mathrm{Gy}$.

\section{Discussion}

In this study of a secondary analysis of a multicenter phase III study, we presented a novel approach for approximating the effective radiation dose to immune cells in circulating blood (abbreviated EDIC). Our results demonstrated an overall inverse relationship between the EDIC and survival outcome in OS and LPFS in a large cohort of patients enrolled in RTOG 617, which is the largest study to date of RT dose response in stage III NSCLC. Multivariable analysis demonstrated that this correlation remained significant after adjustment for other known prognostic factors including tumor dose and GTV, while heart and lung doses were no longer significantly associated with OS after adjusting for these factors. These results support our hypothesis that a high radiation dose to the host immune system, particularly to immune cells in the circulating blood, may impair antitumor immunity, worsening tumor control and survival. This study appears to provide a reasonable explanation for the unexpected worse OS and LPFS from higher dose in patients enrolled the RTOG 0617 trial, suggesting that radiation-induced immunosuppression, rather than radiation-induced grade- 5 cardiac/pulmonary toxicity, may account for the poorer OS in the high-dose arm. It should also be noted that only a few grade- 5 toxicity cases were reported in the RTOG 0617 trial [6].

High lung and heart RT doses were reported to have significant correlations with decreased OS $[23,24]$, which seemed to support the assumption that lung and heart toxicity had contributed to the worse survival seen in the high-dose arm of RTOG 0617. However, our results on actually received doses showed that high MLD and MHD were significantly correlated with OS only under univariate analyses, not in the multivariable Cox model. MLD was significantly correlated with only PFS, while MHD was significant for LPFS under the same multivariable models, suggesting that MLD or MHD is associated with survival due to disease control/progression rather than toxicity. Furthermore, in RTOG 0617, the high-dose arm had less pulmonary toxicity than the low-dose arm, the total reported grade $3+$ pulmonary toxicity was less than $5 \%$, and there were only a few pulmonary deaths and no reported cardiac deaths [6]. Therefore, the potential correlation of lung and heart doses to survival is likely not directly due to conventional heart and lung toxicity. Rather, they may be surrogates of radiation dose to immune structures, such as circulating immune cells in blood and other components of the immune system, which are critical for tumor control. For example, MLD could also be a surrogate of doses to resident lymphocytes in lung tissue and to pulmonary lymph nodes. The significance of this study is the development of the EDIC model, and the validation of its independent correlation with OS in 456 patients with NSCLC. This EDIC model (first presented in ASTRO 2017) was also adopted and validated externally in 117 patients with non-small-cell lung cancer [25], 92 patients with esophageal cancer treated with neoadjuvant chemoradiation [26] and 488 patients with esophageal cancer treated with concurrent chemoradiation [27]. 
One may also ask if the EDIC effect could be a result of tumor volume, which is a potential confounder, since larger tumors are considered to be associated with worse survival. However, our multivariable analysis accounts for this potential confounder by showing that the EDIC was still significantly associated with OS and LPFS after adjusting for GTV size effects. Furthermore, GTV was no longer a significant adverse prognostic factor when it was analyzed together with the EDIC. This result suggests that tumor volume is not likely to explain our observations. It is understandable that the EDIC is a combined effect of tumor volume and "goodness" of the RT plan, i.e., patients with relative small tumors may have a greater EDIC if the plan was not optimized or the tumor was in a special location. The greater significance of the EDIC over GTV suggests the importance of RT planning. Additionally, our data also showed a significant effect of grade $\geq 3$ esophagitis on OS, PFS and LPFS after adjusting for GTV size effects. Esophagitis would certainly not cause tumor progression. However, severe esophagitis may indicate that the patient is more radiosensitive, and a more radiosensitive patient may have a greater risk of severe radiation-induced immunosuppression and thus an increased risk of tumor progression and poorer survival. Completion of the entire chemotherapy course was also a significant factor for OS, PFS and LPFS. Of the total 456 patients, 58 patients did not complete the chemotherapy course. These patients might be not able to tolerate the treatment. It is understandable that they had a worse treatment outcome.

The key clinical implication of this study is that circulating immune cells may be considered as a critical organ at risk during external-beam RT, and that the EDIC is a potentially useful parameter for plan optimization to limit incidental toxicity to circulating immune cells during RT. Several approaches have the potential to reduce the EDIC, including the following: (1) reduction in circulating blood exposure via hypofractionated treatment regimens and/or decrease in the radiation delivery time, (e.g., with very high-dose-rate techniques such as FLASH RT) [28]; (2) optimization of plans by adjustment of beam energies and directions, number of beams, and collimator margins, as well as the use of intensity-modulated radiotherapy (IMRT) and other advanced planning techniques; (3) the use of advanced RT technology, such as image-guided adaptive therapy [29] and proton therapy [30]; and (4) dose de-escalation and/or margin reduction, tailored to individual radiosensitivity [31].

The present study has limitations. Firstly, it did not have blood count data to show correlation of the EDIC with the lymphocyte count. However, the lymphocyte count may not well reflect the radiation killing of immune cells in blood as well as the potential anti-tumor immunity, because lymphocytes can be replenished from the spleen and bone marrow, and there are many subpopulations, such as B cells, T cells, CD8, CD4, CD4 helper, CD4 regulator, NK cells, memory cells, as well as proliferating and non-proliferating cells for each subset in the lymphocytes. These cells have a large variation of radiosensitivity [32]. Russ et al. showed that a very low-dose $(0.01 \sim 0.1 \mathrm{~Gy})$ total body irradiation (TBI) in rats reduced the lymphocyte count to $50 \%$ of the baseline level in hours, and the lymphocyte count recovered to the baseline level several days later [33]. They also showed that multiple low-dose TBIs with weeks interval increased lymphocyte count several times higher than the baseline level, and these rats accepted syngeneic tumor implants (poor immunity), while the rats with lymphocytes recovery to the baseline level rejected syngeneic tumor implants (good immunity). These data support that the lymphocyte count after radiation does not necessary correlate with the radiation dose, and a higher lymphocyte count does not mean a better anti-tumor immunity. In addition, So et al. and Xu et al. have used the EDIC model to study its association with lymphopenia and OS in esophageal cancers $[26,27]$. Both studies found a significant association of the EDIC with lymphopenia. More importantly, they found that the EDIC was much more predictive of OS and distant metastasis-free survival than the lymphopenia [27].

Secondly, the EDIC model was based on relatively simple assumptions, including the following: (1) integral dose was used to approximate the mean dose to the large vessels and small vessels/capillaries; (2) an approximate model was used to calculate the EUD 
contribution to small vessels and capillaries in other organs; (3) the percentages of blood volume assigned to lung, heart, great vessels, and small vessels/capillaries may not reflect the actual blood distribution in the body; (4) average rather than patient-specific parameters were used for approximating the dose contributions; (5) the ITDV did not exclude the lung and heart volumes. These assumptions would affect the accuracy of the model. For example, the great vessels contain a high concentration of lymphocytes. However, our study was initially a proof of concept study. In addition, we expect that more precise values for these parameters would not significantly improve the accuracy of the EDIC model, because the EDIC model is relatively accurate and reliable for its main contributors: the lung and heart. While the EDIC is the estimation of the physical dose to the circulating immune cells in blood, it can also be considered as a parameter that optimally combines three independent factors, the MLD, MHD and ITDV. Additionally, it is notable that this study did not account for the contribution of the other immune substructures such as lymph nodes, lymphatic ducts (particularly the thoracic duct), thymus, bone marrow, and resident lymphocytes in lung and tumor tissue. Future work shall develop and validate a more comprehensive model, including carefully determination of doses to large vessels, lymphatic organs, and resident immune cells in lung and tumor.

\section{Conclusions}

This study demonstrated that the EDIC is significantly associated with local progressionfree survival and overall survival in a large prospective cooperative group study of concurrent chemoradiation for the treatment of unresectable stage III NSCLC. These findings suggest that radiation dose to the immune cells is a critical determinant of treatment outcomes. While an external validation study is needed, the knowledge gained from the present study may be used to guide RT planning to improve tumor control and survival in patients with locally advanced NSCLC.

Supplementary Materials: The following are available online at https:/ /www.mdpi.com/article/10 .3390 / cancers13246193/s1, Table S1: Patient characteristics and their effects on survival.

Author Contributions: Conceptualization, F.-M.K. and J.-Y.J.; methodology, J.-Y.J. and S.G.E.; software, J.-Y.J.; validation, J.-Y.J., F.-M.K. and C.H.; formal analysis, C.H. and R.P.; investigation, Y.X. and H.Z.; resources, S.E.S., J.A.B., M.C.D., V.S.K., S.N., P.I., C.R., J.S.G., C.K. and H.C.; data curation, J.-Y.J., C.H. and R.P.; writing-original draft preparation, J.-Y.J. and F.-M.K.; writing—review and editing, F.-M.K. and S.G.E.; visualization, H.C. and R.P.; supervision, J.D.B., H.C., M.M. and W.C.; project administration, J.D.B., M.M. and W.C.; funding acquisition, F.-M.K. and W.C. All authors have read and agreed to the published version of the manuscript.

Funding: This project was supported in parts by grants U10CA180868 (NRG Oncology Operations), U10CA180822 (NRG Oncology SDMC), UG1CA189867 (NCORP), KQTD20180411185028798, R01CA142840 (PI: Kong) from the National Cancer Institute (NCI) and a grant from Eli Lilly.

Institutional Review Board Statement: This study was conducted according to the guidelines of the Declaration of Helsinki, and approved by the Institutional Review Board of accrual institutions for RTOG 0617 trial.

Informed Consent Statement: Informed consent was obtained from all subjects involved in this study (RTOG 0617 trial).

Data Availability Statement: The data presented in this study are available on reasonable request from the corresponding author and NRG Oncology.

Conflicts of Interest: The authors declare no conflict of interest.

\section{Appendix A}

The following steps are used to estimate the dose to the immune cells in circulating blood: (1) first consider the contribution from a single blood-containing organ; (2) use the blood-flow continuity principle to calculate the dose volume to the blood for a single fraction for a single organ; (3) determine the fractionation effect using a similar approach 
reported by Yovino et al. [18]; (4) convert the blood dose volume data into an equivalent uniform dose (EUD); (5) sum the EUDs for all irradiated organs; (6) define the EUD sums as the effective dose to immune cells in circulating blood.

We assume that a blood-containing organ has $A \%$ of cardiac output (total blood flow) branching into the organ (out of the total blood from the heart) and $B \%$ blood volume contained in the organ at a moment in comparison to the total body blood volume. The time of one blood circulation is $T$, the irradiation time is $t$, the number of RT fractions is $n$, and the organ has received a mean organ dose of MOD. For each fraction, using the blood-flow continuity principle, the blood volume $(V \%)$ and dose $(d)$ contributed by the organ is:

$$
\begin{gathered}
V \%=B \%+(A \%-B \%) * t / T \\
d=\left(\frac{M O D}{n}\right) *\left(\frac{B}{V}\right)
\end{gathered}
$$

Then, we consider the fractionation effect. After each fraction of irradiation, as proposed by Yovino et al. [18], the irradiated blood is uniformly redistributed. Therefore, after the second fraction, the total blood volume can be divided into 3 parts, with each part receiving a dose of $2 * d, 1 * d$, and $0 * d$, respectively. Similarly, after $i$ th fraction, the total blood volume can be divided into $(i+1)$ parts, with each part receiving a dose of $i * d,(i-1) * d, 1 * d$ and $0 * d$, respectively. Assuming the blood volume receiving a dose of $j * d$ after $i$ th fraction is $V(i, j)$, the differential dose volume histogram (DVH) after the $i$ th fraction can be derived by calculating $V(i, j)$ using the following iteration algorithm.

After the 1st fraction, $V(1,1)=V \% * V(0,0)$, and

$$
V(1,0)=(1-V \%) * V(0,0)
$$

After the 2nd faction, $V(2,2)=V \% * V(1,1), V(2,1)=V \% * V(1,0)+(1-V \%) *$ $V(1,1)$ and

After the $n$th fraction,

$$
V(2,0)=(1-V \%) * V(1,0)
$$

$V(n, n)=V \% * V(n-1,1), V(n, n-1)=V \% * V(n-1, n-2)+(1-V \%) * V(n-1, n-1)$,
$V(n, 1)=V \% * V(n-1,0)+(1-V \%) * V(n-1,1)$, and $V(n, 0)=(1-V \%) * V(n-1,0)$

We then convert the DVH into an EUD. Figure A1a shows how EUD varies with $n$ for various $V \%$ for an example organ with $B \% * M O D=3 \mathrm{~Gy}$. It shows that when $V \%>20 \%$ and $n>25$, EUD is almost a constant and can be accurately and simply computed as

$$
E U D=B \% * M O D
$$

Otherwise, EUD is approximated as

$$
E U D=B \% * M O D * k_{1} *\left(\frac{n}{k_{2}}\right)^{\frac{1}{2}}
$$

where $k_{2}$ is approximately 45 , and $k_{1}$ is a dose losing factor due to a low $V \%$. When $V \%=6 \%, k_{1}$ is approximately $85 \%$.

We have estimated $A \%$ and $B \%$ for the major organs in the blood circulation system (Figure A1b) [34]. The organs/components that are irradiated in thoracic RT mainly include (1) lung, (2) heart, (3) large vessels, and (4) small vessels and capillaries in other organs, such as esophagus, muscles, bones, and skin. $A \%$ is $50 \%$ for each lung, $100 \%$ for heart, $>30 \%$ for large vessels, and $5-10 \%$ for other blood vessels if we combine all the small vessels into one organ. $B \%$ is approximately $12 \%$ for lung, $8 \%$ for heart, and $45 \%$ for large vessels, and $35 \%$ for small vessels. Considering that we have $T \sim 1 \mathrm{~min}, t>1 \mathrm{~min}$, according Equation (A1-1), we have $V \% \sim A \%$. Because $n=30-37$, EUD can be reliably calculated as $B \% * M O D$ for lung, heart and large vessels. For the 4 th component (small vessels and capillaries in other organs), we estimated that $V \%=6-8 \%$ Thus, $k_{1} \sim 0.85$, and EUD can be estimated using Equation (A3-2). We further assume that the vessels and capillaries are 
uniformly distributed in the body, so that the average integral total dose (ITD) can be used to replace the MOD for the large vessels and small vessels. Because the CT images did not include the entire body, while $B \%$ for the large and small vessels were for the whole body, we assume that each patient has a weight of 140 pounds, corresponding to $63 \mathrm{Kg}$ or a volume of $61.8 \times 10^{3} \mathrm{CC}$. Thus, a factor of $V_{\text {scan }} / 61.8 \times 10^{3}$ was used to adjust $B \%$ for large vessels and small vessels. The EDIC for thoracic RT can be considered as the sum of EUDs for the four organs/components. Thus, the final EDIC is expressed as:

$$
E D I C=12 \% * M L D+8 \% * M H D+\left[45 \%+35 \% * 0.85 *\left(\frac{n}{45}\right)^{\frac{1}{2}}\right] * \operatorname{ITDV} /\left(61.8 * 10^{3}\right)
$$

where ITDV is the integral total dose volume, which is ITD $* V_{\text {scan }}$.

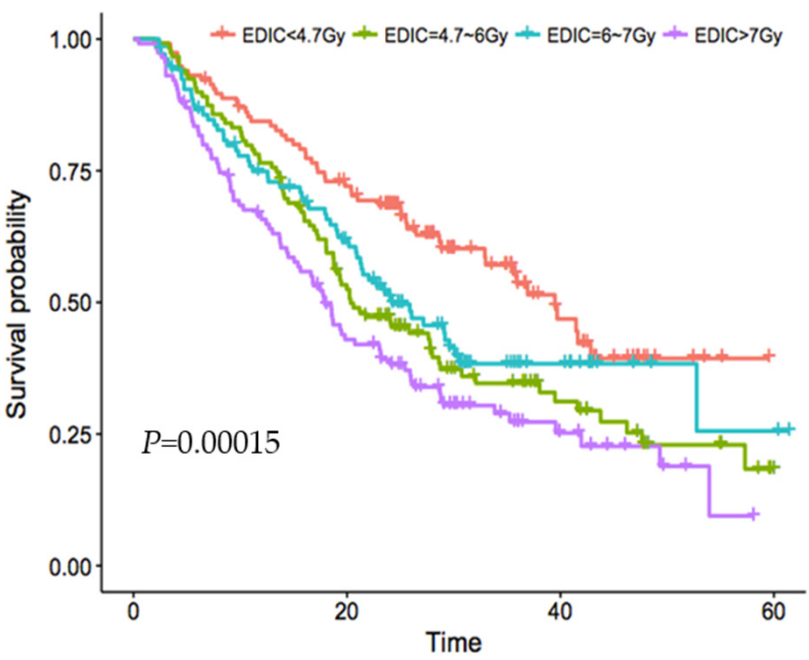

(a)

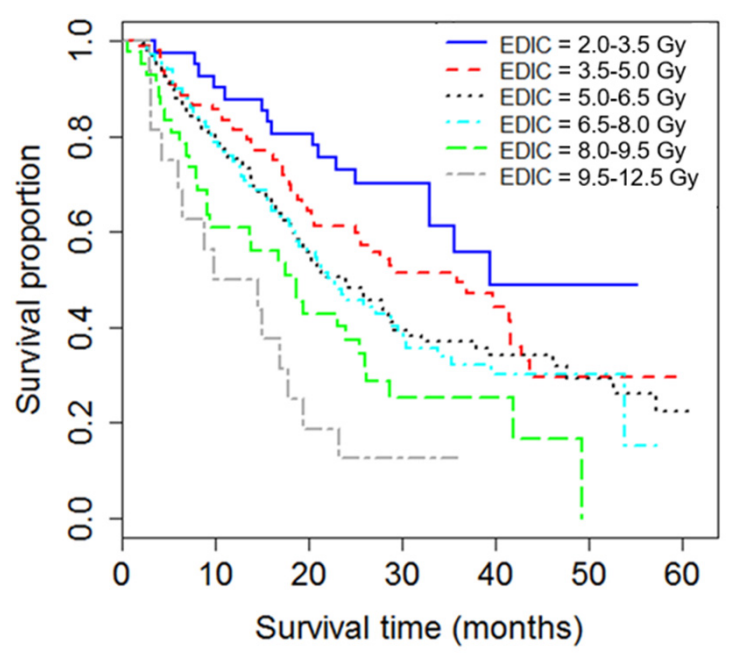

(b)

Figure A1. Computation of the EDIC model. (a) Equivalent uniform dose (EUD) to the total blood varies with fraction number $(n)$ for various $\mathrm{V} \%$ (the percentage blood volume irradiated in a specific organ during each fraction), which contains the $B \%$ of blood volume that receives irradiation with a mean organ dose (MOD). We have $M O D * B \%=3 \mathrm{~Gy}$ for this example organ. $V \%$ depends on the cardiac output $A \%$, blood volume $\mathrm{B} \%$, blood circulation time $T$, and beam-on time $t$ for each fraction and can be determined as $V \%=\mathrm{B} \%+(A \%-\mathrm{v} \%) * t / T$. We note that when $n>20$ and $V \%>20 \%$, $E U D \sim M O D * B \%$. Generally, EUD can be approximated as EUD MOD $* B \% * k_{1} *(n / 45)^{1 / 2}$ for other organs with a smaller $V \%$, where $k_{1}$ is a dose effectiveness factor. (b) Major organs in the blood circulation system and their esti-mated percentage cardiac output $(A \%)$ and percentage blood volume $(B \%)$ based on an anatomy/physiology text book. For thoracic irradiation, 4 organs/components, including lung, heart, great vessels, and small vessels/capillaries in other organs, contribute to the total blood dose. Because $A \% \geq 40 \%$ for lung, heart and great vessels, and with $t \sim 1 \mathrm{~min}$ and $T \sim 1 \mathrm{~min}$, we have $V \%=A \% \geq 40 \%$, and the contribution of dose to the blood for these organs can be estimated to be $E U D \sim M O D * B \%$.

\section{References}

1. Miller, K.D.; Siegel, R.L.; Lin, C.C.; Mariotto, A.B.; Kramer, J.L.; Rowland, J.H.; Stein, K.D.; Alteri, R.; Jemal, A. Cancer treatment and survivorship statistics, 2016. CA Cancer J. Clin. 2016, 66, 271-289. [CrossRef]

2. Tyldesley, S.; Boyd, C.; Schulze, K.; Walker, H.; MacKillop, W.J. Estimating the need for radiotherapy for lung cancer: An evidence-based, epidemiologic approach. Int. J. Radiat. Oncol. Biol. Phys. 2001, 49, 973-985. [CrossRef]

3. Furuse, K.; Fukuoka, M.; Kawahara, M.; Nishikawa, H.; Takada, Y.; Kudoh, S.; Katagami, N.; Ariyoshi, Y. Phase III study of concurrent versus sequential thoracic radiotherapy in combination with mitomycin, vindesine, and cisplatin in unresectable stage III non-small-cell lung cancer. J. Clin. Oncol. 1999, 17, 2692. [CrossRef]

4. Antonia, S.J.; Villegas, A.; Daniel, D.; Vicente, D.; Murakami, S.; Hui, R.; Kurata, T.; Chiappori, A.; Lee, K.H.; De Wit, M.; et al. Overall survival with durvalumab after chemoradiotherapy in stage III NSCLC. N. Engl. J. Med. 2018, 379, 2342-2350. [CrossRef] [PubMed] 
5. Kong, F.-M.; Haken, R.T.; Schipper, M.J.; Sullivan, M.A.; Chen, M.; Lopez, C.; Kalemkerian, G.P.; Hayman, J.A. High-dose radiation improved local tumor control and overall survival in patients with inoperable/unresectable non-small-cell lung cancer: Long-term results of a radiation dose escalation study. Int. J. Radiat. Oncol. 2005, 63, 324-333. [CrossRef] [PubMed]

6. Bradley, J.D.; Paulus, R.; Komaki, R.; Masters, G.; Blumenschein, G.; Schild, S.; Bogart, J.; Hu, C.; Forster, K.; Magliocco, A.; et al. Standard-dose versus high-dose conformal radiotherapy with concurrent and consolidation carboplatin plus paclitaxel with or without cetuximab for patients with stage IIIA or IIIB non-small-cell lung cancer (RTOG 0617): A randomised, two-by-two factorial phase 3 study. Lancet Oncol. 2015, 16, 187-199. [CrossRef] [PubMed]

7. Formenti, S.C. Silvia Formenti on the promise of combining radiotherapy and immunotherapy to treat cancer. Oncology 2016, 30, 289-292. [PubMed]

8. Lee, Y.; Auh, S.L.; Wang, Y.; Burnette, B.; Meng, Y.; Beckett, M.; Sharma, R.; Chin, R.; Tu, T.; Weichselbaum, R.R.; et al. Therapeutic effects of ablative radiation on local tumor require CD8+ T cells: Changing strategies for cancer treatment. Blood 2009, 114, 589-595. [CrossRef]

9. Vanpouille-Box, C.; Formenti, S.C.; Demaria, S. Towards precision radiotherapy for use with immune checkpoint blockers. Clin. Cancer Res. 2017. [CrossRef] [PubMed]

10. Postow, M.A.; Callahan, M.K.; Barker, C.A.; Yamada, Y.; Yuan, J.; Kitano, S.; Mu, Z.; Rasalan, T.; Adamow, M.; Ritter, E.; et al. Immunologic correlates of the abscopal effect in a patient with melanoma. N. Engl. J. Med. 2012, 366, 925-931. [CrossRef]

11. Grossman, S.A.; Ye, X.; Lesser, G.; Sloan, A.; Carraway, H.; Desideri, S.; Piantadosi, S. Immunosuppression in patients with high-grade gliomas treated with radiation and temozolomide. Clin. Cancer Res. 2011, 17, 5473-5480. [CrossRef]

12. Campian, J.L.; Ye, X.; Brock, M.; Grossman, S.A. Treatment-related lymphopenia in patients with stage III non-small-cell lung cancer. Cancer Investig. 2013, 31, 183-188. [CrossRef]

13. Davuluri, R.; Jiang, W.; Fang, P.; Xu, C.; Komaki, R.; Gomez, D.R.; Welsh, J.; Cox, J.D.; Crane, C.H.; Hsu, C.C.; et al. Lymphocyte nadir and esophageal cancer survival outcomes after chemoradiation therapy. Int. J. Radiat. Oncol. Biol. Phys. 2017, 99, 128-135. [CrossRef] [PubMed]

14. Wild, A.T.; Ye, X.; Ellsworth, S.G.; Smith, J.A.; Narang, A.K.; Garg, T.; Campian, J.; Laheru, D.A.; Zheng, L.; Wolfgang, C.L.; et al. The association between chemoradiation-related lymphopenia and clinical outcomes in patients with locally advanced pancreatic adenocarcinoma. Am. J. Clin. Oncol. 2015, 38, 259-265. [CrossRef] [PubMed]

15. Wild, A.T.; Herman, J.M.; Dholakia, A.S.; Moningi, S.; Lu, Y.; Rosati, L.M.; Hacker-Prietz, A.; Assadi, R.K.; Saeed, A.M.; Pawlik, T.M.; et al. Lymphocyte-sparing effect of stereotactic body radiation therapy in patients with unresectable pancreatic cancer. Int. J. Radiat. Oncol. Biol. Phys. 2016, 94, 571-579. [CrossRef]

16. Meyer, K.K. Radiation-induced lymphocyte-immune deficiency. A factor in the increased visceral metastases and decreased hormonal responsiveness of breast cancer. Arch. Surg. 1970, 101, 114-121. [CrossRef] [PubMed]

17. MacLennan, I.C.; Kay, H.E. Analysis of treatment in childhood leukemia. IV. The critical association between dose fractionation and immunosuppression induced by cranial irradiation. Cancer 1978, 41, 108-111.

18. Yovino, S.; Kleinberg, L.; Grossman, S.A.; Narayanan, M.; Ford, E. The etiology of treatment-related lymphopenia in patients with malignant gliomas: Modeling radiation dose to circulating lymphocytes explains clinical observations and suggests methods of modifying the impact of radiation on immune cells. Cancer Investig. 2013, 31, 140-144. [CrossRef]

19. Arina, A.; Beckett, M.; Fernandez, C.; Zheng, W.; Pitroda, S.; Chmura, S.J.; Luke, J.J.; Forde, M.; Hou, Y.; Burnette, B.; et al. Tumor-reprogrammed resident T cells resist radiation to control tumors. Nat. Commun. 2019, 10, 1-13. [CrossRef]

20. Jin, J.; Hu, C.; Xiao, Y.; Zhang, H.; Ellsworth, S.; Schild, S.; Bogart, J.; Dobelbower, M.; Kavadi, V.; Narayan, S.; et al. Higher radiation dose to immune system is correlated with poorer survival in patients with stage III non-small cell lung cancer: A secondary study of a phase 3 cooperative group trial (NRG oncology RTOG 0617). Int. J. Radiat. Oncol. Biol. Phys. 2017, 99, S151-S152. [CrossRef]

21. Harre, J.F.E.; Lee, K.L.; Pollock, B.G. Regression models in clinical studies: Determining relationships between predictors and response. J. Natl. Cancer Inst. 1988, 80, 1198-1202. [CrossRef]

22. Grambsch, P.; Therneau, T. Proportional hazards tests and diagnostics based on weighted residuals. Biometrika 1994, 81, 515-526. [CrossRef]

23. Tucker, S.L.; Liu, A.; Gomez, D.; Tang, L.L.; Allen, P.; Yang, J.; Liao, Z.; Grosshans, D. Impact of heart and lung dose on early survival in patients with non-small cell lung cancer treated with chemoradiation. Radiother. Oncol. 2016, 119, 495-500. [CrossRef]

24. Speirs, C.K.; DeWees, T.A.; Rehman, S.; Molotievschi, A.; Velez, M.A.; Mullen, D.; Fergus, S.; Trovo, M.; Bradley, J.D.; Robinson, C.G. Heart dose is an independent dosimetric predictor of overall survival in locally advanced non-small cell lung cancer. $J$. Thorac. Oncol. 2017, 12, 293-301. [CrossRef] [PubMed]

25. Ladbury, C.J.; Rusthoven, C.G.; Camidge, D.R.; Kavanagh, B.D.; Nath, S.K. Impact of radiation dose to the host immune system on tumor control and survival for stage III non-small cell lung cancer treated with definitive radiation therapy. Int. J. Radiat. Oncol. 2019, 105, 346-355. [CrossRef]

26. So, T.H.; Chan, S.K.; Chan, W.L.; Choi, H.; Chiang, C.L.; Lee, V.; Lam, T.C.; Wong, I.; Law, S.; Kwong, D.; et al. Lymphopenia and radiation dose to circulating lymphocytes with neoadjuvant chemoradiation in esophageal squamous cell carcinoma. Adv. Radiat. Oncol. 2020, 5, 880-888. [CrossRef]

27. Xu, C.; Jin, J.-Y.; Zhang, M.; Liu, A.; Wang, J.; Mohan, R.; Kong, F.-M.; Lin, S.H. The impact of the effective dose to immune cells on lymphopenia and survival of esophageal cancer after chemoradiotherapy. Radiother. Oncol. 2020, 146, 180-186. [CrossRef] 
28. Jin, J.-Y.; Gu, A.; Wang, W.; Oleinick, N.L.; Machtay, M.; Kong, F.-M. Ultra-high dose rate effect on circulating immune cells: A potential mechanism for FLASH effect? Radiother. Oncol. 2020, 149, 55-62. [CrossRef]

29. Kong, F.-M.; Haken, R.K.T.; Schipper, M.; Frey, K.A.; Hayman, J.; Gross, M.; Ramnath, N.; Hassan, K.A.; Matuszak, M.; Ritter, T.; et al. Effect of midtreatment PET/CT-adapted radiation therapy with concurrent chemotherapy in patients with locally advanced non-small-cell lung cancer: A phase 2 clinical trial. JAMA Oncol. 2017, 3, 1358-1365. [CrossRef] [PubMed]

30. Chang, J.Y.; Verma, V.; Li, M.; Zhang, W.; Komaki, R.; Lu, C.; Allen, P.K.; Liao, Z.; Welsh, J.; Lin, S.H.; et al. Proton beam radiotherapy and concurrent chemotherapy for unresectable stage III non-small cell lung cancer: Final results of a phase 2 study. JAMA Oncol. 2017, 3, e172032. [CrossRef] [PubMed]

31. Jin, J.-Y.; Wang, W.; Haken, R.T.; Chen, J.; Bi, N.; Sadek, R.; Zhang, H.; Lawrence, T.S.; Kong, F.-M. Use a survival model to correlate single-nucleotide polymorphisms of DNA repair genes with radiation dose-Response in patients with non-small cell lung cancer. Radiother. Oncol. 2015, 117, 77-82. [CrossRef] [PubMed]

32. Heylmann, D.; Badura, J.; Becker, H.; Fahrer, J.; Kaina, B. Sensitivity of CD3/CD28-stimulated versus non-stimulated lymphocytes to ionizing radiation and genotoxic anticancer drugs: Key role of ATM in the differential radiation response. Cell Death Dis. 2018, 9, 1053. [CrossRef] [PubMed]

33. Russ, S.; Chambers, H.; Scott, G.; Mottram, J. Experimental studies with small doses of X-rays. Lancet 1919, $193,692-695$. [CrossRef]

34. Tortora, G.J.; Derrickson, B.H. Principles of Anatomy and Physiology, 14th ed.; Wiley: New York, NY, USA, 2013. 